\title{
Defeating the Digital Divide
}

\section{Executive Summary}

As the world becomes increasingly reliant on the internet, from online schooling to working from home, broader and higher quality access has never been more important. However, expanding internet infrastructure presents a unique challenge in terms of cost, economic efficiency, and capacity requirements. Our team aims to optimize the process of improving connectivity by predicting the price of bandwidth over the next 10 years, calculating bandwidth needs for a variety of household scenarios, and determining the best distribution of cellular nodes over a given region.

We first predicted future internet costs in the United States(US) and the United Kingdom(UK) by using random forest regression, a supervised machine learning algorithm that combines the predictions of multiple decision trees to give accurate forecasts. The model was trained on a dataset of 48 mainland US states and their respective population densities, cost of living, average download speeds, and average price per Mbps. After achieving a reasonable root mean square error of 0.660 , the random forest was then applied to the entirety of the US and UK. The population densities and cost of living were adjusted based on the expected percent change over 10 years, and a regressed exponential function was used to determine future average download speeds. These features served as input into the random forest which then predicted the future price. The model ultimately predicted a decrease of $\$ 0.23$ per Mbps in the US and $\$ 0.57$ in the UK over the next decade, which proved to be a sensible result.

Next, we calculated the bandwidth demands for various household scenarios and determined the bandwidth required to satisfy $90 \%$ and $99 \%$ of predicted demand. Each scenario involved a household with a hypothetical weekly schedule of activities and information based on age, education, and job status. We created a simulation of a typical week with 112 waking hours and determined the bandwidth usage for each hour where the probability of a person performing the activity was proportional to the percent of total time needed for the task. For each scenario, the maximum bandwidth was recorded over 1000 trials, and the 90th and 99th percentile of the distribution was found. 14.5 Mbps and 15.5 Mbps were sufficient for $90 \%$ and $99 \%$ coverage for scenario 1, $20.8 \mathrm{Mbps}$ and $21.8 \mathrm{Mbps}$ for scenario 2, and 20.6 Mbps and 21.9 Mbps for scenario 3.

Finally, we developed a model that optimally distributes $4 \mathrm{G}$ and $5 \mathrm{G}$ cellular nodes in arbitrary regions. We studied the comparative advantages and disadvantages of each network type - surmising that while $4 \mathrm{G}$ networks are often discounted, its $5 \mathrm{G}$ counterparts offer fast speeds at shorter signal ranges. To calculate the locations for placing the nodes, we calculated the center of mass by integrating mass with respect to position for each region. We also determined that cities eligible for $5 \mathrm{G}$ networks should have population densities of 777 people per square mile or higher and an annual household income of at least $\$ 103,689.32$. Combining these two techniques yielded an efficient distribution plan for cellular nodes. 


\section{Contents}

1 Executive Summary 250

2 Part I: The Cost of Connectivity 253

2.1 Restatement of the Problem . . . . . . . . . . . . . . . . . . 253

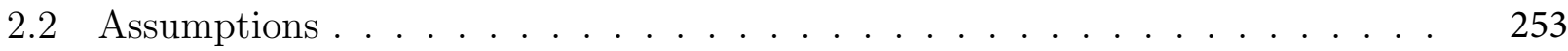

2.3 Model Development . . . . . . . . . . . . . . . . . . . . . 253

2.3 .1 Factor Identification . . . . . . . . . . . . . . . 253

2.3.2 Random Forest Regression . . . . . . . . . . . . . . . . . . . 254

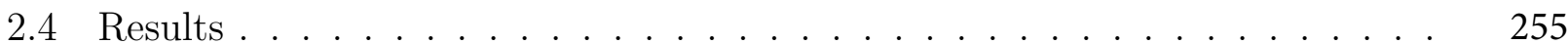

2.5 Strengths and Weaknesses . . . . . . . . . . . . . . . . . . 255

3 Part II: Bit by Bit 256

3.1 Restatement of the Problem . . . . . . . . . . . . . . . . . 256

3.2 Assumptions . . . . . . . . . . . . . . . . . . . . 256

3.3 Model Development . . . . . . . . . . . . . . . . . . . . . . 257

3.3.1 Determining the Hours Each Person Spends on the Internet . . . . . 257

3.3.2 Simulating Outcomes . . . . . . . . . . . . . . . 258

3.4 Results . . . . . . . . . . . . . . . . . . . . 258

3.5 Strengths and Weaknesses . . . . . . . . . . . . . . . . . . 260

4 Part III: Mobilizing Mobile 260

4.1 Restatement of the Problem . . . . . . . . . . . . . . . . . . 260

4.2 Assumptions . . . . . . . . . . . . . . . . . . . 260

4.3 Model Development . . . . . . . . . . . . . . . . . . . . . 261

4.4 Results . . . . . . . . . . . . . . . . . . . . . . 264 
4.5 Strengths and Weaknesses . . . . . . . . . . . . . . . . . . 267

$\begin{array}{llr}5 & \text { Conclusion } & 267\end{array}$

5.1 Further Studies . . . . . . . . . . . . . . . . . . . . . . . . 267

5.2 Summary . . . . . . . . . . . . . . . . . . . . . . 267

$\begin{array}{llr}6 & \text { References } & 269\end{array}$

$\begin{array}{llr}7 & \text { Appendix } & 270\end{array}$

7.1 Part 1: The Cost of Connectivity . . . . . . . . . . . . . . . . . 270

7.2 Part 2: Bit by Bit . . . . . . . . . . . . . . . . 271 


\section{Part I: The Cost of Connectivity}

\subsection{Restatement of the Problem}

In this problem, we are tasked with predicting the cost per unit of bandwidth(Mbps) over the next decade for consumers in the United States and the United Kingdom.

\section{$2.2 \quad$ Assumptions}

1. There will be no significant policy changes regarding standards for internet speed, cost, and availability in the next 10 years. Due to the unpredictable nature of new legislation and government initiatives, we are unable to account for these potential changes in our modeling.

2. The amount of internet provided in Mbps per US dollar will be constant regardless of how much money is spent. The majority of internet users will not spend enough money such that they begin experiencing diminishing returns for each dollar spent. Economics of scale are therefore not applicable to individual households.

3. Future internet usage and cost trends in the UK are comparable to US states and depend on the same factors. The United States and the United Kingdom have similar internet infrastructure and geographical trends such as population density (e.g. the two countries contain both high and low population density areas).

4. The population density and cost of living index in a given area increase by $1.31 \%$ and 2.20\% respectively, which are sensible rates for the United States. The percent increase in population density in California was found to be $1.31 \%$ according to the PPIC[1]. California has a large land area and contains a relatively uniform mix of urban and rural areas. As a result, its overall increase in population density can likely be generalized to the United States. The average increase in the cost of living index can be modeled by the inflation rate[2]. Since we are looking at the change of the index over a long period of time (10 years), it is reasonable to use a constant average inflation rate per year in order to model the growth of the cost of living over a decade. .

\subsection{Model Development}

\subsubsection{Factor Identification}

The population density is a relevant factor given the cost discrepancies between urban and rural areas. For a sparsely populated region, the price of internet is significantly higher for an individual consumer because fewer people are available to recuperate the fixed infrastructure cost. On the other hand, the utility obtained per unit of internet infrastructure is greater in densely populated areas where more consumers are available to handle the expenses. Thus, 
we incorporate the population density data of mainland US states[3] into our model along with the expected annual change of 1.31

Next, the cost of living indicates the amount of money required to achieve a certain standard of living in a given area. With a higher cost of living, the dollar price for goods and services, including the internet, will also be greater. Therefore, we utilize a cost of living index[4] that compares a state's cost of living with the average for the United States. For example, a state with an index value of 110 compared to the mean of 100 in the US has a $10 \%$ higher cost of living. We utilize the average change in the index to model the internet costs for the next 10 years while accounting for inflation.

Lastly, we include the average download speed for each state[5] to ensure that the model accounts for any potential trends between the factor and internet cost. For example, it is possible that areas with high download speed have well-established infrastructure and therefore have cheaper internet. We also created two exponential regression models based on US and the UK download speed trends[6] to predict the values over the next 10 years as shown in Figure 1. These predictions are then used as part of the overall regression model.
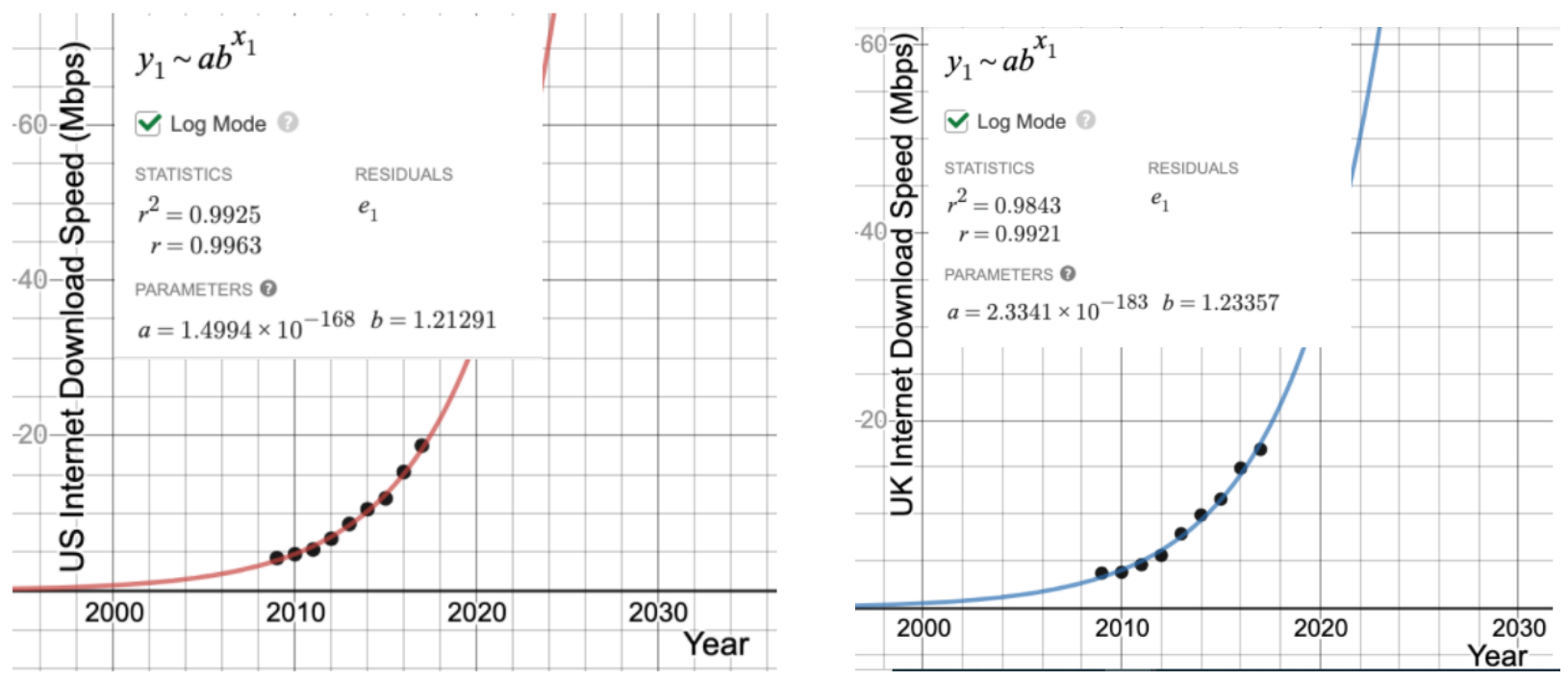

Figure 1: Exponential Regression Models

\subsubsection{Random Forest Regression}

To predict internet cost over time, we used the Scikit-learn Python library to train a random forest regression model on the factors described above. This supervised machine learning model employs an ensemble of decision trees to make an accurate and robust prediction. Each decision tree is trained on a random subset of the dataset, shown in Figure 2, to reduce intercorrelation and utilizes algorithms to determine optimal logical splits. Following the created splits, data can be categorized and a regression output is produced. The outputs of the individual decision trees are then averaged for the final ensemble prediction. Our 
random forest model consists of 20 decision trees, each limited to a max depth of five nodes to improve generalization to unseen data.

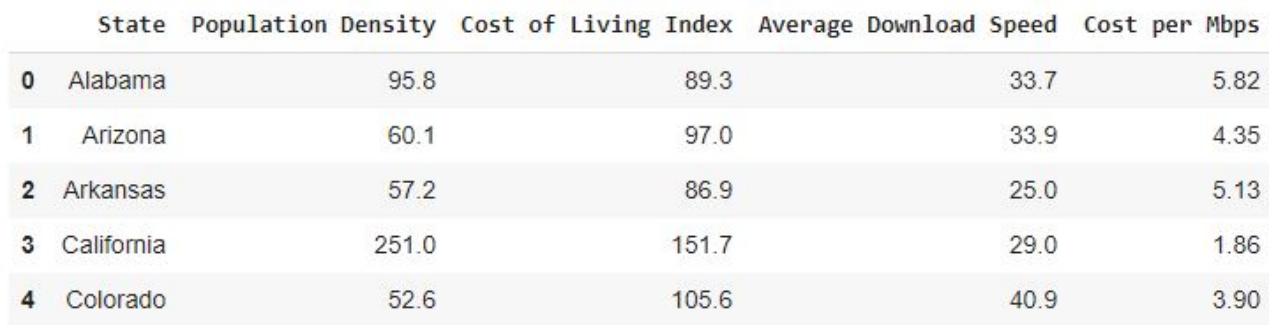

Figure 2: First few rows of the training data

The random forest regressor was first trained on the set of 48 US states using the features of population density, cost of living index, and average download speed. The target column consisted of the state's average internet cost[7] in dollars per Mbps. After training, the model was used to predict the cost of the internet for the US and UK over the next 10 years. This was accomplished by making predictions for each country's average feature values and then applying the appropriate annual change to each input for the next prediction. The population density was increased by $1.31 \%$, the cost of living index by $2.20 \%$, and the average download speed by the amount indicated by the exponential regressions. By performing this process over 10 iterations, we were able to find the predicted future internet costs for users in the two countries.

\subsection{Results}

The random forest regression achieved a root mean square error (RMSE) of 0.660 when predicting the internet costs for US states. This value represents a typical difference between the predicted and actual internet prices in US dollars, which is an acceptable error given the magnitude of the prices.

The 10-year US and UK regressions produce the trends shown in Figure 3. Although there are some deviations, the model predicts a decrease of $\$ 0.57$ (from $\$ 4.33$ to $\$ 3.76$ ) in the average price per Mbps in the US and a decrease of $\$ 0.57$ (from $\$ 3.18$ to $\$ 2.61$ ) in the UK. As expected, the price of internet access decreases over time which can be attributed to increased population density and improved infrastructure. Overall, the predictions are consistent with the expected results.

\subsection{Strengths and Weaknesses}

The random forest model allows us to effectively combine multiple factors to make a prediction. The algorithm is robust to skewed, non-linear data which makes it ideal for processing features such as population density. Furthermore, the ensembling process minimizes the risk of overfitting, a prevalent issue where a machine learning model learns the training data 

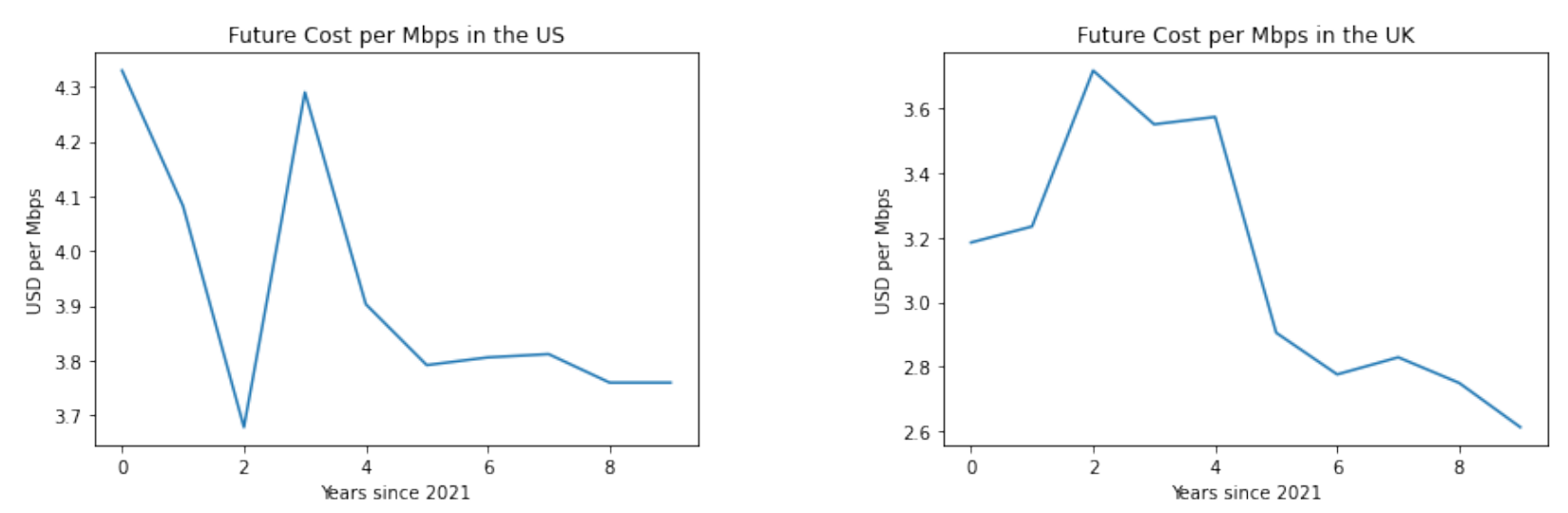

Figure 3: Projected cost of bandwidth in Mbps for the US and the UK

too closely and later fails to accurately predict unseen examples. However, a weakness of random forest regression is the fact that it is not easily interpretable since it consists of a large number of decision trees. It is difficult to pinpoint the exact decision process that the algorithm undergoes to arrive at its prediction.

\section{Part II: Bit by Bit}

\subsection{Restatement of the Problem}

In this problem, we are tasked with finding what minimum bandwidth households would need to cover their internet usage $90 \%$ and $99 \%$ of the time. Three scenarios were given:

1. A couple in their early 30's (one is looking for work and the other is a teacher) with a 3-year-old child.

2. A retired woman in her 70's who cares for two school-aged grandchildren twice a week.

3. Three former M3 Challenge participants sharing an off-campus apartment while they complete their undergraduate degrees full-time and work part-time.

\subsection{Assumptions}

1. We assume that each day and week will have the same probabilities. Each day and week in the year will overall be extremely similar so we ignore any special circumstances.

2. We observe that each day's usage (Sunday vs. Saturday vs. Monday etc.) does not differ substantially, which resultantly solves the issue of the grandma caring for her grandchildren. Beyond the grandmother, the aforementioned assumption also resolves situational occurrences for each person, both now and in the future. 
3. The people in the given situations are in the US and not the UK. The data provided is only relevant for the US.

4. People will always use best quality streaming for Youtube (HD) and Netflix (Ultra HD), even if they only "need" less e.g. $1 \mathrm{mb} / \mathrm{s}$ for $S D$ quality, they will never use it at that quality so we will assume that the bandwidth needed will be at the higher quality. SD quality usually is unsatisfactory and people generally want to use the best quality for the best experience if it's available.

5. Each pre-college school day is 5 hours, giving a total of 25 hrs/week. Every class will also be on a video conference format. Since a general school day is around 7 hours, we made a general estimate that the amount of actual video-conferencing and internet usage would come to around 5 hours.

6. College students also leverage online platforms for their classes in the wake of a pandemic. Their classes span an average of $16 \mathrm{hrs} /$ week, via video conferencing softwares [8]. In light of the additional time that college students have in a remote academic setting, it is safe to say that they will consciously look to take a more rigorous - albeit longer - college course load, leading us to take the higher bound of the class hrs/week (16 hrs).

7. Teachers' work hours are the same as students (5 hours) and they do not work after school hours. Justification: Due to the variability in each day for a teacher -inclusive of meetings and personal commitments - it's difficult to pinpoint their daily commitment to school-related curricula. As a result, we went ahead and assumed that they do not provide after school help, for the most consistency in results.

8. People are awake to use the internet 16 hours a day. 8 hours is a recommended amount of time for an average person to sleep[9].

9. People only access the internet through one medium at a time and do not multitask. Most people generally only consume the internet through one source at a time. Furthermore, this is a simplifying assumption that allows us to run the simulation and assign each person a specific task and internet consumption for a specific hour.

\subsection{Model Development}

\subsubsection{Determining the Hours Each Person Spends on the Internet}

Each person was first assigned a list of digital activities they would perform in a week. Each activity had attributes like time spent, lower bound of bandwidth usage, and upper bound of bandwidth usage. Age group, education level, and employment status were all factors used to determine this list of digital activities[6]. The age group was the first categorization to associate values with the ages of the people for the scenario. Utilizing the data provided[6], we were able to determine internet tendencies for entertainment purposes depending on the 
age of the user. This included the amount of time they spent watching videos, listening to audio, etc. Next, in order to account for the global shift to remote learning and work, we added hours of schooling to those applicable, namely people in the age groups of 2-11, 12-17, and 18-34. Similarly, teachers must work through online, remote instruction and encounter similar internet usage as their students. As a result, school related activities were added on top of entertainment activities that were already present in the list of digital activities.

For employment, those in part-time jobs were considered to be in-person workers because remote part-time jobs are generally hard to obtain, especially for undergraduate students. As a result, jobs were not added onto the list of internet activities as they do not contribute to household bandwidth consumption.

\subsubsection{Simulating Outcomes}

Finally, we simulated the bandwidth demand for each scenario for every single hour for an entire week. Given that people are awake around 16 hours a day, we simulated $7 \cdot 16=112$ hours of internet usage. For each hour, the probability that a person would be performing a given internet activity is proportional to the percentage of time they spend on that activity over the entire week. For example, if a user spends 25 hours a week in school out of 112 hours, they would have a probability of $25 / 112$ or $22.3 \%$ chance of attending school for any of the 112 hours. These random tasks would be selected for each person in the simulation and the individual bandwidth demand would be calculated. If the activity had a higher and lower bound to bandwidth usage, the actual bandwidth usage is determined by uniformly selecting a random floating point number between the bounds of the bandwidth usage. The individual bandwidth is then summed up in order to find the total household bandwidth demand for that given hour. This process is then repeated for each waking hour in the week.

We repeated the simulation 1000 times for every single scenario, representing 1000 weeks of internet usage for each scenario. In each week/trial, we recorded the maximum bandwidth demanded, since a household would require a bandwidth greater than the maximum bandwidth demanded at every single hour in order to successfully cover the internet needs for that week. The maximum values for all 1000 weeks were recorded and then sorted in ascending order. Finally, the 90th percentile and the 99th percentile values were calculated from the maximum bandwidth demand distribution. These represent the minimum bandwidth needed to cover $90 \%$ and $99 \%$ of the demand respectively as $90 \%$ of the bandwidth demands are less than the 90th percentile and $99 \%$ of the bandwidth demands are less than the 99th percentile.

\subsection{Results}

By running the program through each scenario 1000 times and plotting the data, we achieved 3 histograms of distributions of the maximum bandwidth needed for a given hour. In these histograms, shown in Figure 4, the vertical orange line represents the required bandwidth 
to meet the total internet demand $90 \%$ of the time and the vertical red line represents the required bandwidth to meet the total internet demand $99 \%$ of the time.
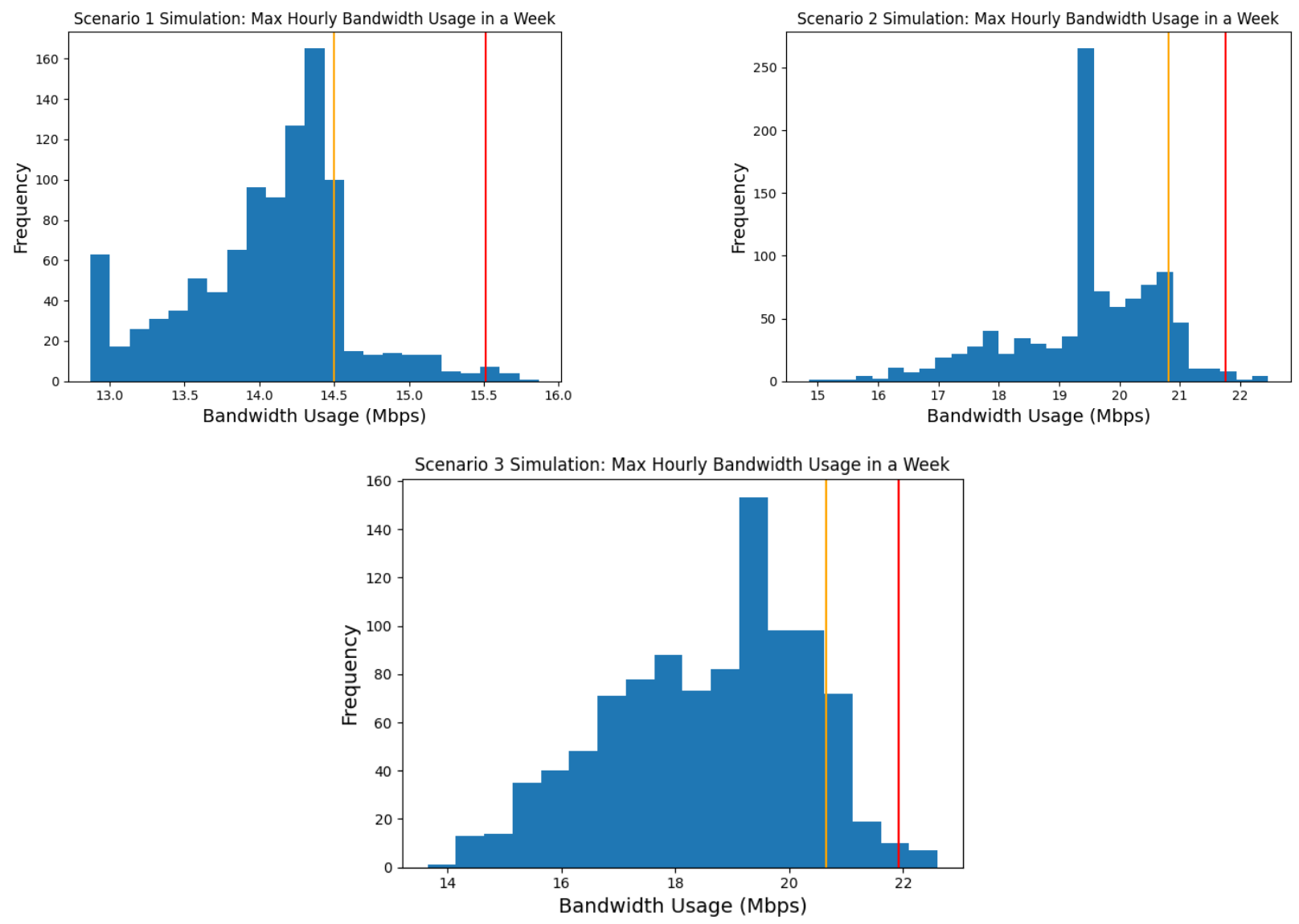

Figure 4: Frequency histograms of the distribution of maximum bandwidth usage for 1000 simulation trials.

Furthermore, as shown in the summarized table, in scenario 1, the required bandwidth to satisfy $90 \%$ of bandwidth demand is $14.5 \mathrm{Mbps}$ and $99 \%$ of demand is $15.5 \mathrm{Mbps}$. In scenario 2, $20.8 \mathrm{Mbps}$ is required for $90 \%$ of demand and $21.8 \mathrm{Mbps}$ is required for $99 \%$ of demand. Finally, in scenario 3, 20.6 Mbps is required for $90 \%$ of demand and $21.9 \mathrm{Mbps}$ is required for $99 \%$ of demand.

\begin{tabular}{|c|c|c|}
\hline Scenario & $90 \%$ Coverage & $99 \%$ Coverage \\
\hline 1 & $14.5 \mathrm{Mbps}$ & $15.5 \mathrm{Mbps}$ \\
\hline 2 & $20.8 \mathrm{Mbps}$ & $21.8 \mathrm{Mbps}$ \\
\hline 3 & $20.6 \mathrm{Mbps}$ & $21.9 \mathrm{Mbps}$ \\
\hline
\end{tabular}

Table 1: Summary of the minimum number of Megabits per Second of internet required to cover internet usage $90 \%$ and $99 \%$ of the time for each of the three scenarios 


\subsection{Strengths and Weaknesses}

This model is accurate at predicting bandwidth usages and the amount of bandwidth required in order to satisfy those demands. All values predicted were between 14-22 Mbps. Our predictions are further supported by the FCC's household broadband guide[10], which recommends a medium service of 12 to $25 \mathrm{Mbps}$ for moderate internet usage in households of 2-3 people. Moreover, this model is very flexible in considering multiple factors and internet usage caused by various internet activities. In the case that more data about internet usage for a new or existing activity comes up, our model and simulation can be easily adjusted to predict given the new factor.

One significant weakness of this model is that the data utilized to calculate the probabilities was from the first quarter of 2020. While the data is not too far off, current internet usage tendencies will be different; currently, the model likely underpredicts the amount of needed bandwidth as people are using digital devices more due to being stuck at home in the midst of the pandemic. Moreover, people usually have activities they will perform at a certain time, like kids going to school from roughly 8 AM to 3 PM, and this model essentially has all activities being able to occur at any given hour interval.

\section{Part III: Mobilizing Mobile}

\subsection{Restatement of the Problem}

In this problem, we are tasked with developing a model for optimally placing cellular nodes in a given region using population, demographic, and bandwidth usage data.

\subsection{Assumptions}

1. The price of $5 G$ internet plans will remain constant across different regions. Due to the small market share of $5 \mathrm{G}$ networks (namely under the domain of Verizon and AT\&T), there's limited offerings for pricing, all of which hover around the $\$ 89$ point according to a GSMA Intelligence report [11]. As a result, it made the most sense to assume that this price is homogeneous across all purchased $5 \mathrm{G}$ plans.

2. The population densities remain constant across the hypothetical subregions created by M3. (eg: the Region A1, Region B3) provided by M3[6]. In short, data set[6] provides information and shapes for the 3 hypothetical regions $\mathrm{A}, \mathrm{B}, \mathrm{C}$ and their respective subregions. While the M3 Data provided the population and area of the subregions in their hypothetical diagram, there is no way to account for fluctuations in population densities across the subregion.

3. A majority of households that have annual incomes $\geq \$ 103,689.32$ and are located in a subregion with a minimum population density of 777 people/sq mile, will naturally 
choose the $5 \mathrm{G}$ plan (these values are derived later on in our approach). With $5 \mathrm{G}$ plans discretely offering faster speeds than their $4 \mathrm{G}$ opponents, a family in the position, both geographically and financially, would reasonably choose to do so.

4. Walls and other natural barriers will not hinder $5 G$ speeds inside the effective signal range. It's nearly impossible to quantify the effects of such obstacles with the data provided[6]. Moreover, the impact will depend on the material composition of the barrier - another difficult metric to model.

\subsection{Model Development}

To optimize both internet connectivity and affordability for any given population, we sought to create an efficacious combination of $4 \mathrm{G}$ and $5 \mathrm{G}$ internet access points - capitalizing on their niche benefits, while mitigating any potential disadvantages. There are three relevant factors that need to be considered when differentiating between $4 \mathrm{G}$ and $5 \mathrm{G}$ networks: signal range, latency, internet speed. The following best characterize the distribution of such factors in the larger framework of the two networks: Simply put, 5G networks offer significantly faster internet speeds, which come at the expense of an abridged signal range. On the other hand, $4 \mathrm{G}$ networks have much more expansive coverage, but operate at higher latency rates, and thereby, slower speeds[12].

Given the more frequent use of $4 \mathrm{G}$ networks, largely due to their feasibility for populations anytime and anywhere, we decided to pinpoint the location of these nodes first in the hypothetical regions provided by the M3 Data[6]. Using principles from packing problem optimization, we created a mathematical model that can be used to place $4 \mathrm{G}$ towers at the points of maximum effectiveness.

Our mathematical model calculates the center of mass for each region, where mass refers to population, using the assumption that the population density is uniformly distributed throughout the region. By utilizing a small tiling of the region, we can approximate the moments of the $\mathrm{x}$-axis and $\mathrm{y}$-axis and obtain the coordinates of the center of mass. By placing the $4 \mathrm{G}$ towers at the centers of mass, we will guarantee that the maximum number of people can effectively utilize the bandwidth it provides. The number of internet nodes in a given region will depend on the area of the region and the effective signal range for the different internet towers.

First, set the origin at the bottom left of the region. While it does not necessarily have to be here, this keeps consistency between different regions. Additionally, let the side length of the square be $\Delta x$. We can approximate $\Delta x$ using any subregion, whichever one is more convenient/has the least amount of area outside of the tiling. After we approximate the area of the subregion in terms of $(\Delta x)^{2}$, since we are given the areas of the subregions, we can approximate $\Delta x$.

As mentioned, we seek the centers of mass of each region. The center of mass, represented 
as $(\bar{x}, \bar{y})$ has coordinates:

$$
\begin{gathered}
\bar{x}=\frac{M_{y}}{M} \\
\bar{y}=\frac{M_{x}}{M}
\end{gathered}
$$

where $M_{x}$ and $M_{y}$ represent the moments about the $x$ and $y$ axis respectively, and $M$ represents the area of the region (given in the data). Thus, in order to obtain the center of mass, we first find the moments. Since we are working under uniform mass density, we can simply let the density function $\delta(x, y)=1$, thus making the moments equivalent to:

$$
\begin{aligned}
& M_{x}=\iint_{D} y d A \\
& M_{y}=\iint_{D} x d A
\end{aligned}
$$

for domain $D$ represents the domain of the moments and area of the region $A$. Since there is no easy way to bound domain $D$ with functions, we instead use a Riemann sums to approximate these integrals. Using the original tiling, we will plot the centers of each square in region 2 . The centers fully contained within the inner boundaries will be used to approximate the moments. For convenience, suppose $P$ is the set of all centers contained within the region. If $P=\left(x_{p}, y_{p}\right)$, then we have that

$$
\begin{aligned}
& M_{x} \approx \Delta A \sum_{(x, y) \in P} y \\
& M_{y} \approx \Delta A \sum_{(x, y) \in P} x
\end{aligned}
$$

Though we can't simplify the summation without knowing our actual region, we can calculate $\Delta A$. By definition, we have that $\Delta A=\Delta x \cdot \Delta y$, but since we are tiling with squares, we have that $\Delta y=\Delta x$ so:

$$
\begin{aligned}
& M_{x} \approx(\Delta x)^{2} \sum_{(x, y) \in P} y \\
& M_{y} \approx(\Delta x)^{2} \sum_{(x, y) \in P} x
\end{aligned}
$$

Calculating out these moments and then dividing by $M$ will yield the coordinates of the center of mass.

Another factor prevalent in the decision making process between $4 \mathrm{G}$ and $5 \mathrm{G}$ networks includes the price tag that each carries; at large, $4 \mathrm{G}$ systems are near ubiquitously cheaper than their 5G counterparts - making household income an important factor in the model. Hence, we decided that there had to be some minimum threshold for determining whether a city should implement $5 \mathrm{G}$ internet nodes.

To encompass the threshold, we opted for a criteria based model, in which a given region must meet both of the following criteria to be eligible for $5 \mathrm{G}$ network implementations. 
The first is a population density $\geq 777$ persons $/$ miles $^{2}$. Since almost all $5 \mathrm{G}$ networks are located in urban centers because of the relatively small range of $5 \mathrm{G}$ signals, we want to ensure that planting a $5 \mathrm{G}$ tower will deliver benefits to the most consumers as possible. Urban clusters (regions that are slightly less packed than cities) are classified as having a population density of 777 people/square mile. Using this data point as a minimum will ensure that we maximize consumer utility. Furthermore, we found the top 10 leading cities with $5 \mathrm{G}$ networks have population densities greater than this value.

The second is an average annual household income $\geq \$ 103,689.32$. We gathered that the average American spends $\$ 57.25$ on internet service plans [13]. The average monthly income in the United States is $\$ 5,555$ [14]. Dividing these values yields that $1.03 \%$ of monthly income is allocated towards an internet plan. Moving onward, we used a constant of $\$ 89$ for the cost of a $5 \mathrm{G}$ plan. This value was derived from the GSMA Intelligence report that found the average $5 \mathrm{G}$ monthly cost in the United States [11]. In turn, this yielded the inequality:

$$
\frac{(\text { Avg Annual Household Income })}{12} \cdot(0.0103)>89 \text {. }
$$

When computed, this outputted a minimum household income $=\$ 103,689.32$ for a family to reasonably afford $5 \mathrm{G}$ service. If a given region meets both criteria, it'll be eligible for a $5 \mathrm{G}$ network in the context of our model. Ideally the web of $5 \mathrm{G}$ nodes should be placed in a central hotspot, the centroid of a population density curve. This will maximize the amount of people the network reaches as well as provide higher connection speeds to a larger number of people.

\begin{tabular}{|c|c|c|c|}
\hline Region & Population Density $\left(\mathrm{ppl} \mathrm{per} \mathrm{mi}^{2}\right)[6]$ & Yearly Income (USD) $[6]$ & 5G Eligibility \\
\hline A1 & 570 & $\$ 27,941.00$ & No \\
\hline A2 & 1778 & $\$ 30,929.00$ & No \\
\hline A3 & 1945 & $\$ 47,163.00$ & No \\
\hline A4 & 168 & $\$ 34,273.00$ & No \\
\hline A5 & 3452 & $\$ 30,425.00$ & No \\
\hline A6 & 650 & $\$ 46,659.00$ & No \\
\hline B1 & 1113 & $\$ 99,652.00$ & No \\
\hline B2 & 486 & $\$ 134,375.00$ & No \\
\hline B3 & 270 & $\$ 173,188.00$ & No \\
\hline B4 & 487 & $\$ 108,306.00$ & No \\
\hline B5 & 331 & $\$ 132,00$ & Yes \\
\hline B6 & 646 & $\$ 214,125.00$ & Yes \\
\hline B7 & 237 & $\$ 104,209.00$ & Yes \\
\hline C1 & 3863 & $\$ 190,729.00$ & Yes \\
\hline C2 & 11600 & $\$ 139,261.00$ & Yes \\
\hline C3 & 10120 & $\$ 152,500.00$ & Yes \\
\hline C4 & 5396 & $\$ 206,875.00$ & \\
\hline C5 & 10069 & & \\
\hline C6 & 3252 & & $\$ 1400$ \\
\hline
\end{tabular}




\begin{tabular}{|l|l|l|l|}
\hline $\mathrm{C} 7$ & 5262 & $\$ 151,731.00$ & Yes \\
\hline
\end{tabular}

Table 2: 5G Eligibility by Subregion

To calculate the coordinates of the $5 \mathrm{G}$ towers, we used the same mass-center approach, essentially applying the findings of the $4 \mathrm{G}$ model to a smaller land-mass. Similarly, the amount of $5 \mathrm{G}$ towers will depend strictly on the area of the given region and the effective signal range distance.

\subsection{Results}

Provided in Figure 5 are the results for $4 \mathrm{G}$ internet node placement for subregion B2 and $5 \mathrm{G}$ internet node placement for subregion $\mathrm{C} 2$.

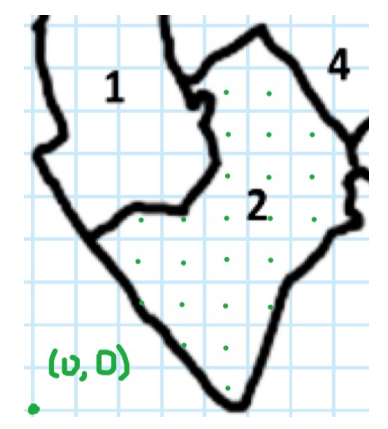

Figure 5: Tiling of Subregion 2 with Origin Marked

As stated in the previous section, we can approximate $\Delta x$ using any subregion within the same region. In this example, we utilize subregion 3, with the area being approximated relatively accurately to $22(\Delta x)^{2}$ as shown in Figure 6: The approximation is calculated by

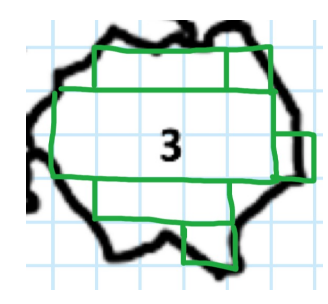

Figure 6: Approximate Tiling of Subregion 3

summing up the 19 outlined tiles and the remaining area in the region that is not contained in either of the outlined tiles, which is roughly equal to 3 full tiles. Since the area of subregion 3 is given to be 4.64 square miles, we can approximate $\Delta x$ to be:

$$
\begin{aligned}
22(\Delta x)^{2} & \approx 4.64 \\
\Delta x & \approx 0.46
\end{aligned}
$$


Now, we look for the center of mass.As aforementioned, in order to obtain the center of mass, we first find the moments. The approximations for the moments are

$$
\begin{aligned}
& M_{x} \approx \Delta A \sum_{(x, y) \in P} y \\
& M_{y} \approx \Delta A \sum_{(x, y) \in P} x
\end{aligned}
$$

for similarly defined set $P$. Using the equivalence we obtained earlier, that is $\Delta A=(\Delta x)^{2}$, we have that

$$
\Delta A=(\Delta x)^{2} \approx .21
$$

Next, we will plug in all $P$, which were marked in Figure 5. All dots have $x$ or $y$ values of form $(2 n-1) \frac{\Delta x}{2}$ for integers $n \geq 1$, so we can multiply these values by the amount of times each value occurs. This gives us the following values for the moments:

$$
\begin{aligned}
& M_{x} \approx(.21)\left(\frac{\Delta x}{2}\right)(1 \cdot 1+3 \cdot 2+5 \cdot 4+7 \cdot 4+9 \cdot 5+11 \cdot 3+13 \cdot 3+15 \cdot 2) \\
& M_{x} \approx(.21)(101 \Delta x) \\
& M_{y} \approx(.21)\left(\frac{\Delta x}{2}\right)(5 \cdot 3+7 \cdot 4+9 \cdot 8+11 \cdot 6+13 \cdot 3) \\
& M_{y} \approx(.21)(110 \Delta x)
\end{aligned}
$$

Now that we have the values of our moments, we can plug these values back in to our original equations for $\bar{x}$ and $\bar{y}$. Given that the mass $M$, which represents the area of the region, 4.35, we obtain the following values:

$$
\begin{aligned}
& \bar{x}=\frac{M_{y}}{M} \approx 2.443 \\
& \bar{y}=\frac{M_{x}}{M} \approx 2.243
\end{aligned}
$$

Thus, for the given tiling, a $4 \mathrm{G}$ tower will be placed at $(2.443,2.243)$.

We will show the similar steps for another example, specifically subregion 2 of Region $\mathrm{C}$ shown in Figure 7, in which a $5 \mathrm{G}$ tower would be placed.

Once again, let the side length of the square be $\Delta x$. Although not shown, we can approximate $\Delta x$ using subregion 3 of Region $\mathrm{C}$, which is approximately the size of a rectangle with side lengths approximately $2.5 \Delta x$ and $8 \Delta x$. Since the area of subregion 3 is given to be 0.1 , we can approximate $\Delta x$ to be:

$$
\begin{aligned}
(2.5 \Delta x)(8 \Delta x) & \approx 0.1 \\
20 \Delta x & \approx 0.1 \\
\Delta x & \approx \sqrt{\frac{1}{200}} .
\end{aligned}
$$




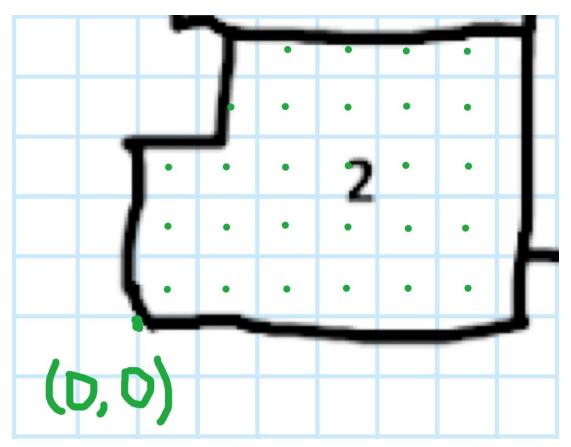

Figure 7: Tiling of Subregion 2

We have the same approximations for the moments:

$$
\begin{aligned}
& M_{x} \approx \Delta A \sum_{(x, y) \in P} y \\
& M_{y} \approx \Delta A \sum_{(x, y) \in P} x
\end{aligned}
$$

Next, plugging in all of the $P$ and substituting in $\Delta A=(\Delta x)^{2}=\frac{1}{200}$ gives us the following values for the moments:

$$
\begin{aligned}
& M_{x} \approx \frac{1}{200}(89 \Delta x) \\
& M_{y} \approx \frac{1}{200}(67 \Delta x)
\end{aligned}
$$

Now that we have the values of our moments, we can plug these values back in to our original equations for $\bar{x}$ and $\bar{y}$. Given that the mass $M$, which represents the area of the region, 0.14, we obtain the following values:

$$
\begin{aligned}
& \bar{x}=\frac{M_{y}}{M} \approx 0.225 \\
& \bar{y}=\frac{M_{x}}{M} \approx 0.169
\end{aligned}
$$

Thus, for the given tiling, a $5 \mathrm{G}$ tower will be placed at $(0.225,0.169)$.

Overall, we can then reproduce the above steps for all of the subsections and regions. For other subsections in the same region, the same tiling dimensions can be used, but for other regions the tiling dimensions will be different. However, the steps can be followed in the exact same pattern just with different values of $\Delta x$. Note that if one wanted to have all of the regions around locations with the same tiling, the coordinates can be converted into square miles and then translated accordingly.

\subsection{Strengths and Weaknesses}

Our model is particularly accurate at determining the optimal point for placing $4 \mathrm{G}$ and $5 \mathrm{G}$ nodes due to our thorough mathematical approach. Moreover, given the rather tedious 
process of establishing $5 \mathrm{G}$ networks, our model is able to filter through cities based on indicators that will determine if the region is a suitable area for $5 \mathrm{G}$ internet.

However, our model does not deem $4 \mathrm{G}$ and $5 \mathrm{G}$ networks in a given region as mutually exclusive; therefore, there is a chance for overlap between the two networks. Families who decide to enroll in the $4 \mathrm{G}$ plan, in spite of qualifying for the $5 \mathrm{G}$ plan, would cause an operational inefficiency in our model.

\section{Conclusion}

\subsection{Further Studies}

Our first model is formed on the basis of a layered decision making tree-encompassing correlations, proportional relationships, and a random forest regression. As a result, it becomes increasingly difficult to identify the exact process that our algorithm followed to eventually arrive at its output. Our second model relies heavily on data procured from the first quarter of 2020 - when COVID-19 was a paramount confounding variable in the internet usage for any given household. With the pandemic creating a new sense of normality, we can expect to see definitive changes in our use of web-based services, and in turn, access to the internet. Our third model doesn't distinguish particular subregions in terms of the type of connectivity $-4 \mathrm{G}$ or $5 \mathrm{G}$ - provided. As a result, households that, under geographic and fiscal circumstances, qualify for $5 \mathrm{G}$ may choose to willingly implement $4 \mathrm{G}$ in their homes, leading to inaccuracies in our initial predictions. Recreating our models to account for and track each factor with specific weighting would greatly improve the accuracy of our results.

\subsection{Summary}

We provided an estimation of the projected internet costs in the US and the UK over the next decade. By first training a machine learning model on factors such as population densities, cost of living, current average download speeds, and current average price per Mbps on data from the continental United States, we were then able to apply the model to the US and UK as a whole. The algorithm ultimately predicted that US prices would drop by $\$ 0.23$ per Mbps and UK prices would drop by $\$ 0.57$ over the next decade.

Next, we utilized the computational capabilities of code to run a simulation with a thousand trials to accurately determine the required bandwidth to cover $90 \%$ and $99 \%$ of internet usage for three scenario households. Using factors like age, education level, and employment status to determine the internet usage tendencies of individuals, we were able to obtain a distribution of maximum bandwidth usage for each household in a week. Looking at the 90th and 99th percentiles allowed us to figure out the minimum bandwidth needed to cover the projected internet usage.

Finally, we developed a method to supply a given area with internet capabilities using 4G 
and 5G towers depending on characteristics of a region. Variables like annual household income and population density allowed us to determine whether a region is suitable for use of $5 \mathrm{G}$ towers, which provides better connection at a greater cost and lower signal radius. In order to place cell nodes in the most optimal locations, the center of mass of each region was found, allowing us to place the fewest $4 \mathrm{G}$ and $5 \mathrm{G}$ towers while maximizing area coverage.

Ultimately, ensuring access to both stable and high-speed internet sources curates a paradigm shift for future generations - in which technical or fiscal disparities don't govern end outcomes for any individual. Investing in the infrastructure to efficiently set up such network capabilities is fundamental to establishing a better tomorrow. With the continued understanding and modeling of disruptive technologies including, but not limited to $5 \mathrm{G}$ towers and bandwidth allocation, we can foster a resilient working class with the tools at their disposal to stand out, conquer obstacles, and reach excellence. 


\section{$6 \quad$ References}

[1] - California's Future: Population. Jan. 2018, www.ppic.org/wp-content/uploads/r118hj2r.pdf.

[2] - Pettinger, Tejvan, et al. "Why Does the Cost of Living Keep Rising?" Economics Help, 20 Oct. 2016, www.economicshelp.org/blog/11826/inflation/why-does-the-costof-living-keep-rising.

[3] - "List of States by Population Density." List of States By Population Density, state.1keydata.com/ state-population-density.php.

[4] - Cost Of Living Index by State 2021, worldpopulationreview.com/state-rankings/costof-living-index-by-state.

[5] - Average Internet Speed by State 2021, worldpopulationreview.com/state-rankings/averageinternet-speed-by-state.

[6] - Defeating the Digital Divide Data, MathWorks Math Modeling Challenge 2021, https://m3challenge.siam.org/node/523.

[7] - Shibu, Sherin. "Here's Where People Shell Out the Most and the Least for Internet." PCMAG, PCMag, 16 Nov. 2020, www.pcmag.com/news/heres-where-people-shell-outthe-most-and-the-least-for-internet.

[8] - "How College Differs from High School." Academic Support Programs — Baylor University, https://www.baylor.edu/support_programs/index.php?id=88158.

[9] - "How Much Sleep Do We Really Need?" Sleep Foundation, 31 July 2020, https://www.sleepfoundation.org/how-sleep-works/how-much-sleep-do-we-really-need.

[10] - "Household Broadband Guide." Federal Communications Commission, 11 Mar. 2020, www.fcc.gov/consumers/guides/household-broadband-guide.

[11] - "Intelligence Brief: How Much Will We Pay for 5G?" Mobile World Live, 13 June 2019, www.mobileworldlive.com/blog/intelligence-brief-how-much-will-we-pay-for-5g.

[12] - Huang, Michelle Yan. "Why You Shouldn't Get Too Excited about 5G Yet." Business Insider, Business Insider, 16 Oct. 2020, www.businessinsider.com/5g-high-speedinternet-cellular-network-issues-switch-2019-4.

[13] - Catherine McNally Sr. Writer, et al. "How Much Is the Average Internet Bill?" Reviews.org, 8 Dec. 2020, www.reviews.org/internet-service/how-much-is-internet.

[14] - "Q: How Much Do Average Jobs Pay per Month in 2021?" ZipRecruiter, www.ziprecruiter.com/Salari Salary-per-Month. 


\section{Appendix}

\subsection{Part 1: The Cost of Connectivity}

q1.py

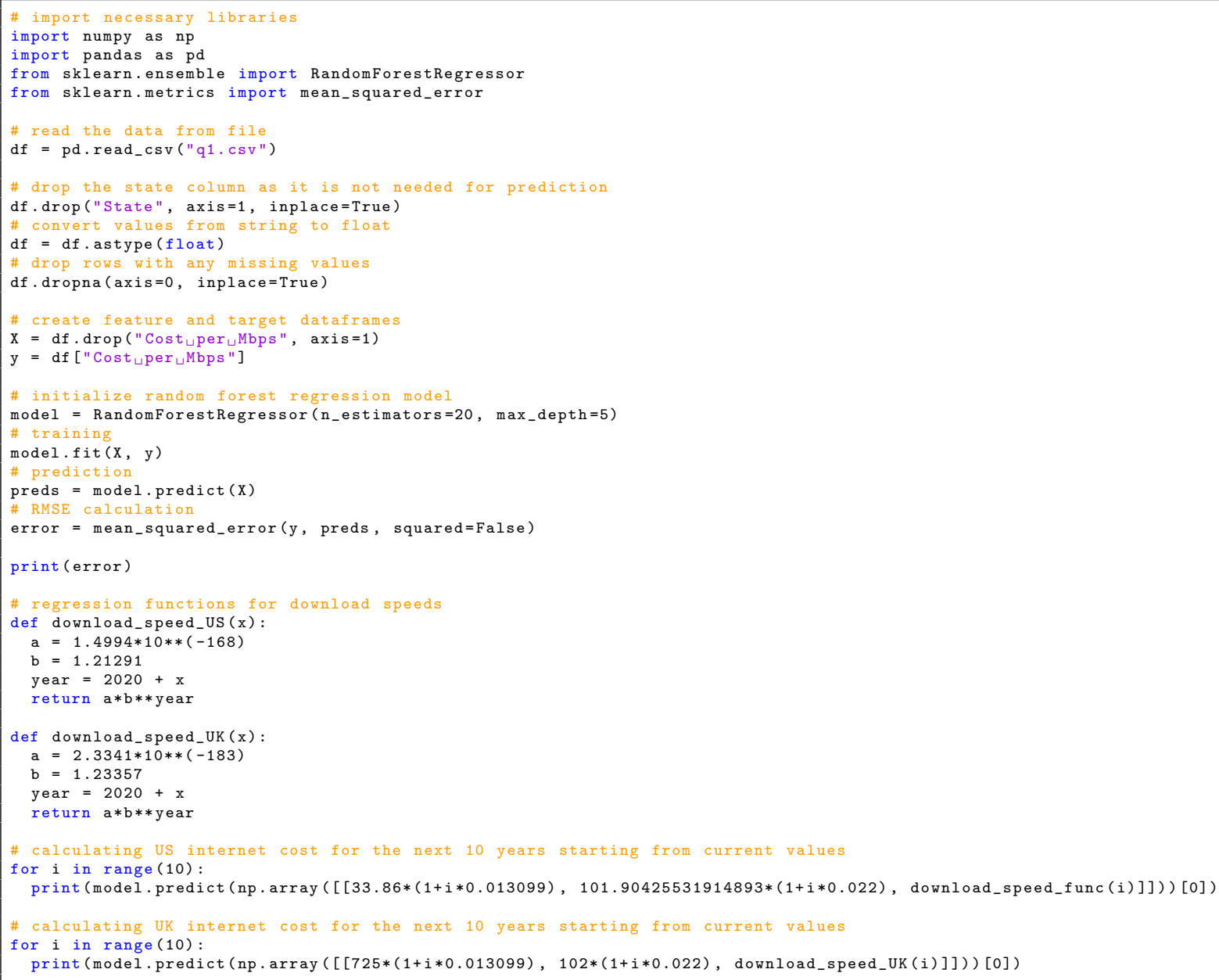




\subsection{Part 2: Bit by Bit}

main.py

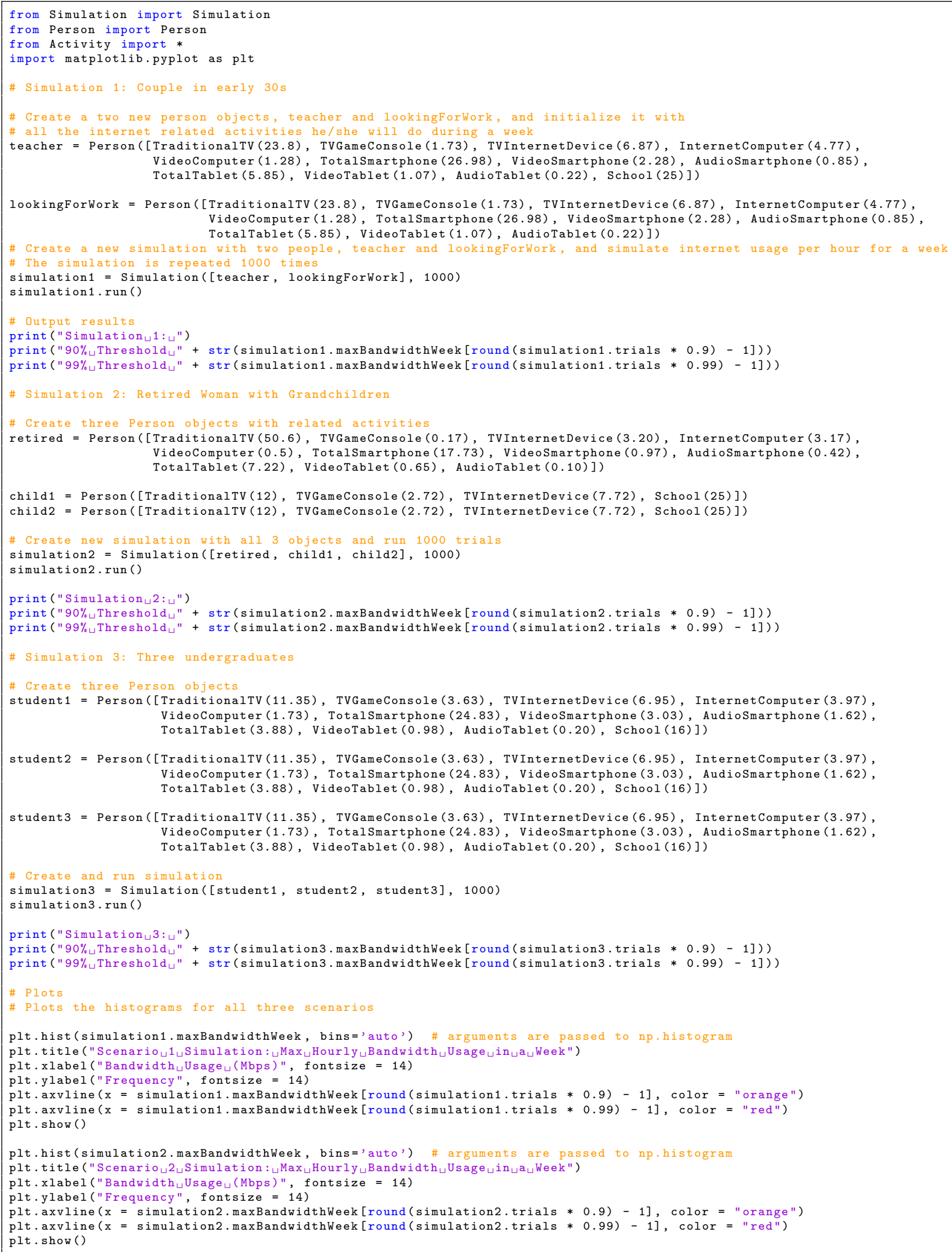




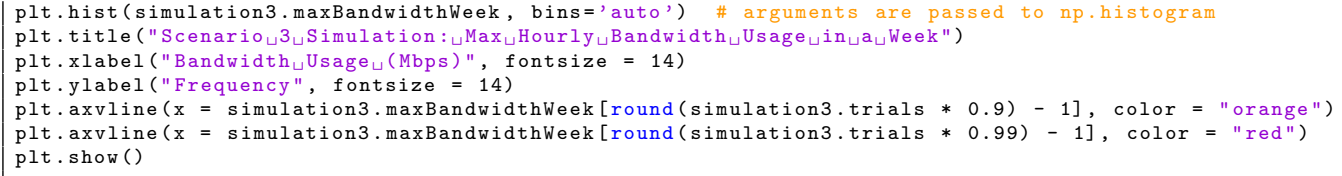

Simulation.py

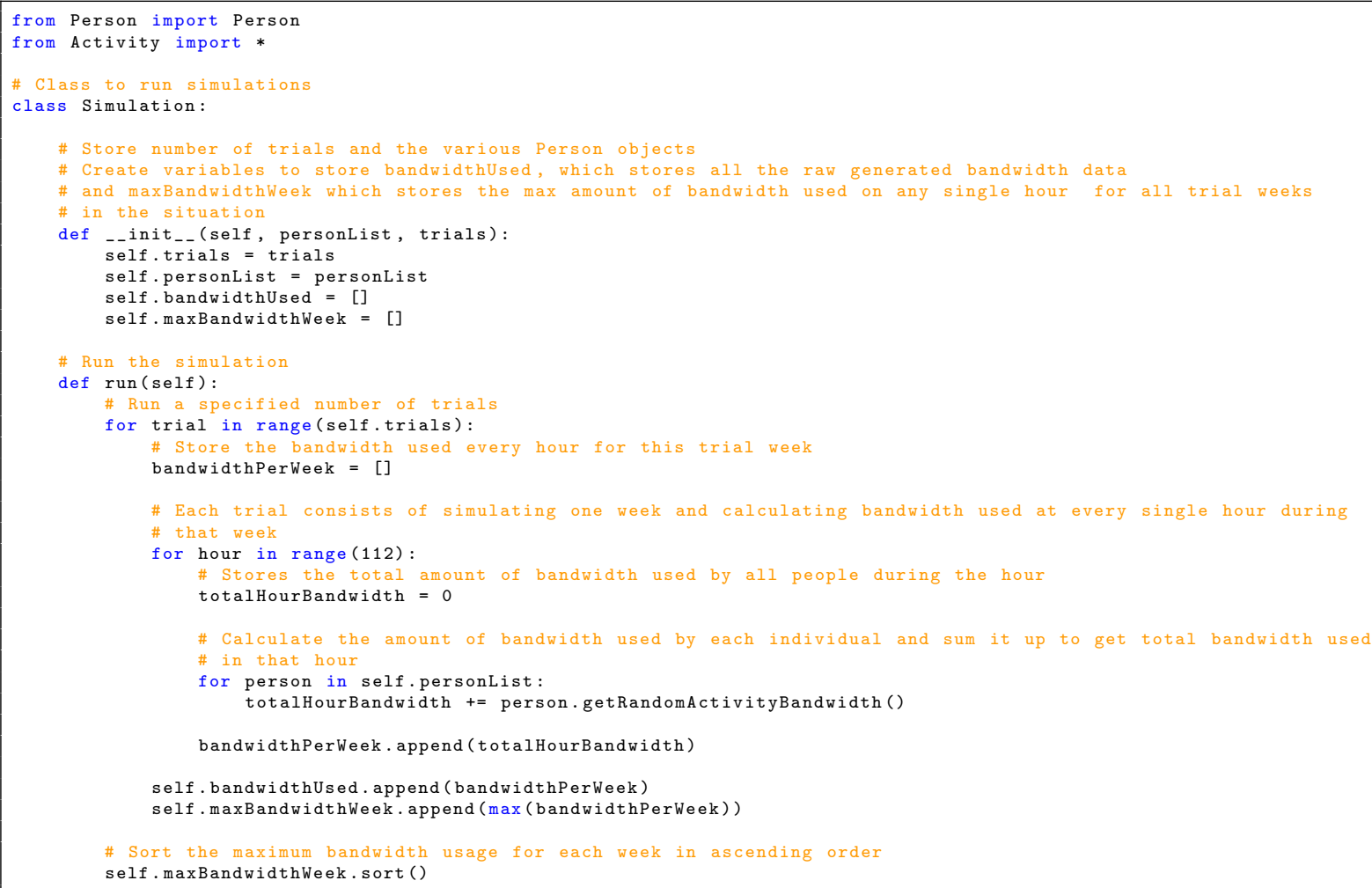

Person.py

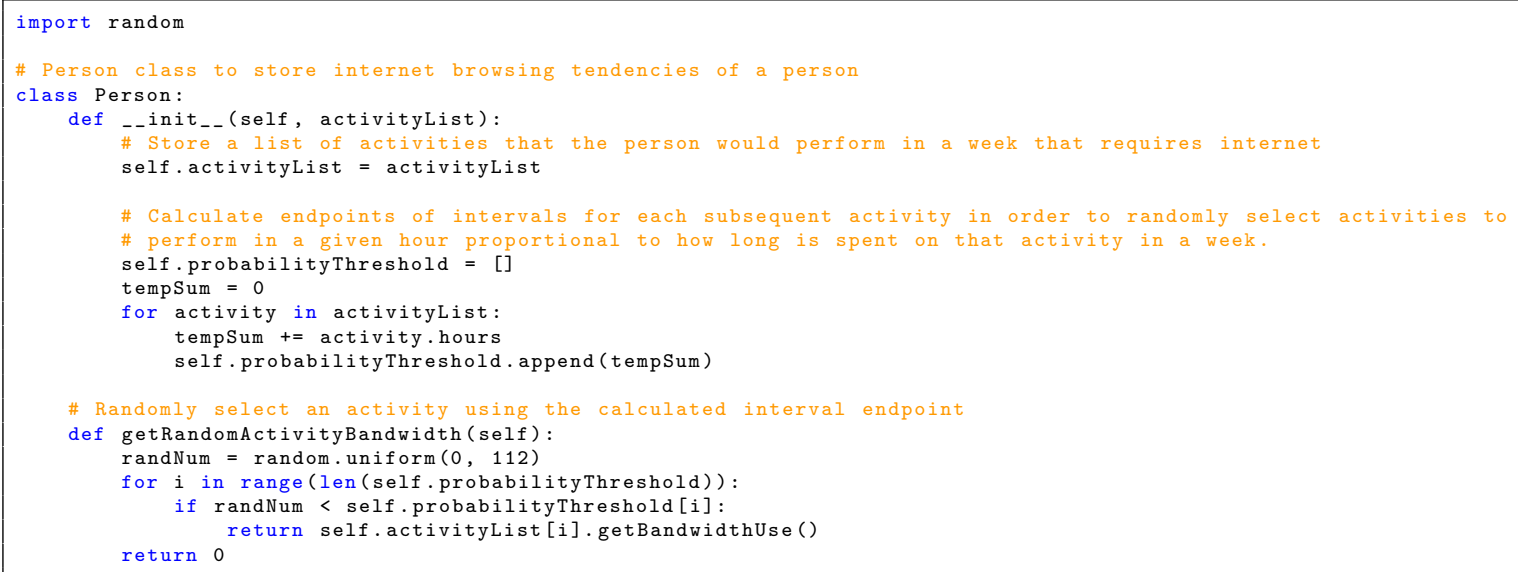




\section{Activity.py}

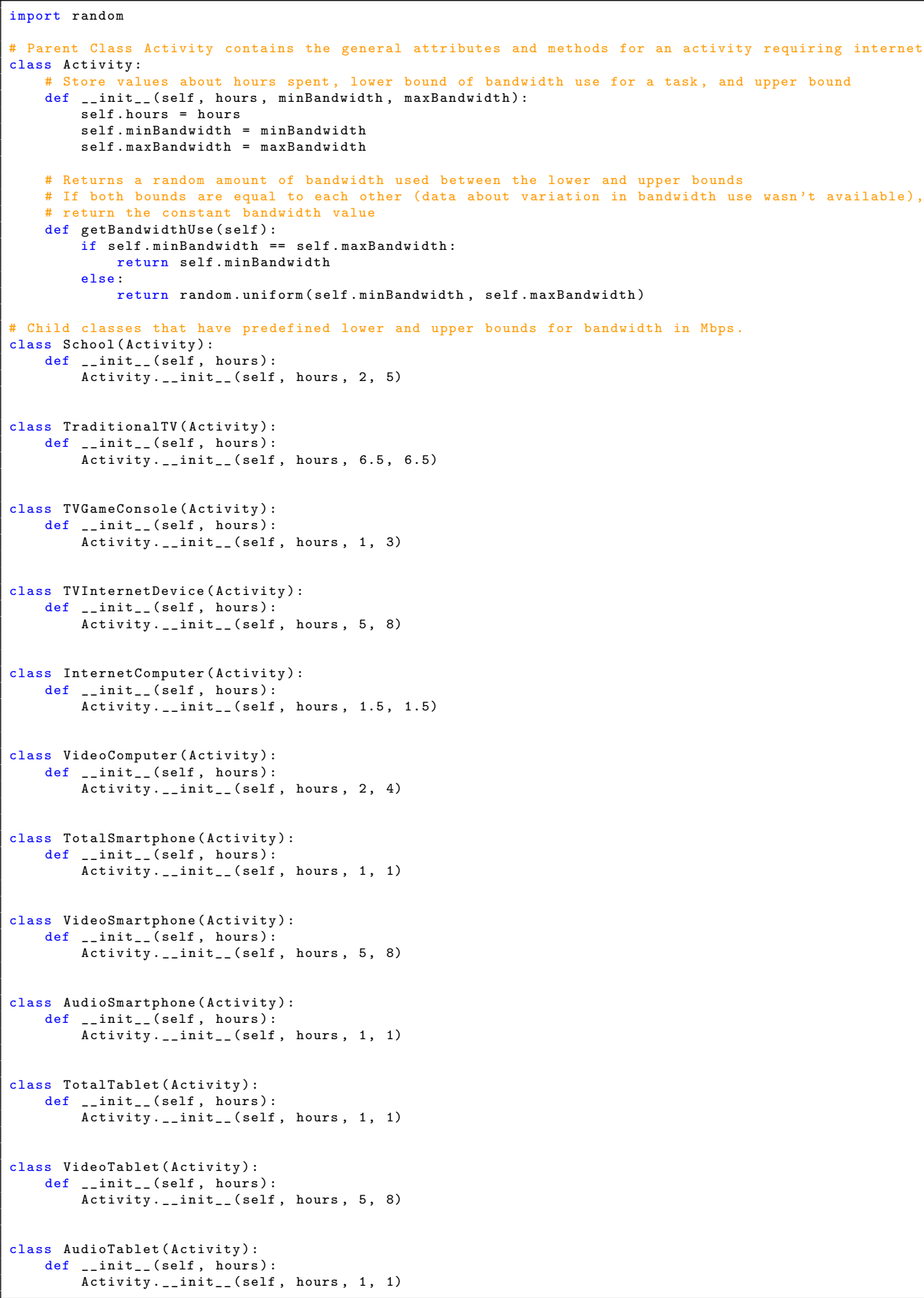

